تأثير مستخلصات صمغ نبات المز .Commiphora sp. والأوراق الخضر اء لنبات الفيكسنتأ Ficus nitida

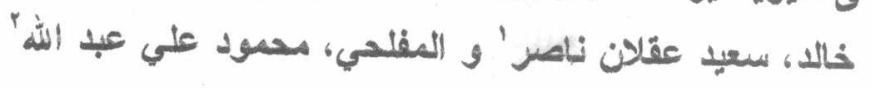

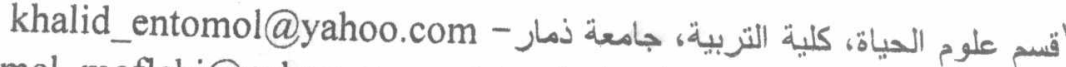

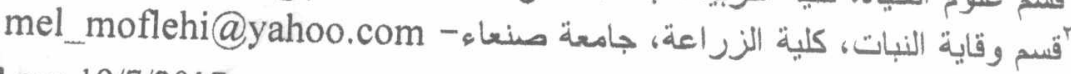

تمت دراسة تأثير كل من المستخلص المائي و الميثانولي والاسيتوني و البنزيني لكل من وناء

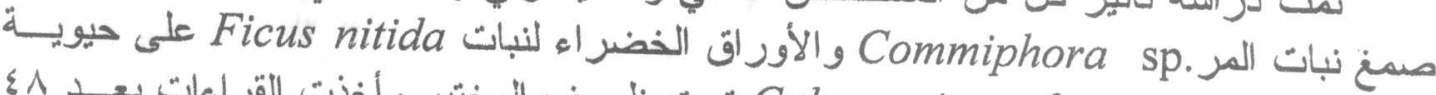

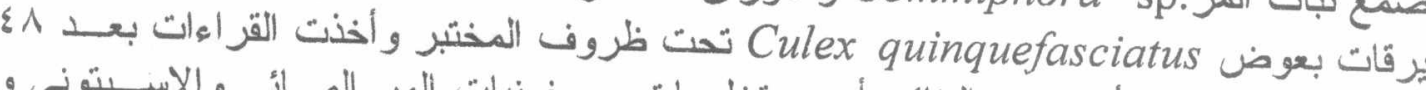

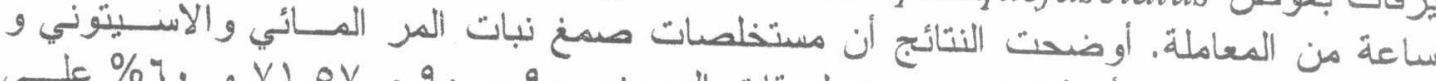

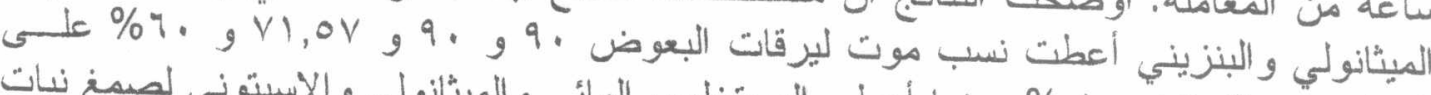

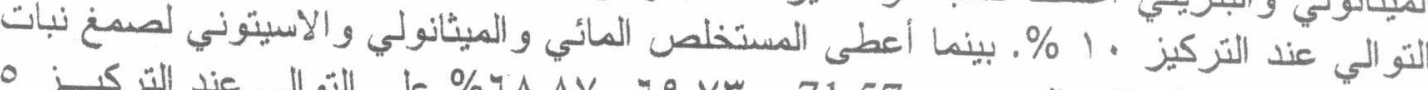

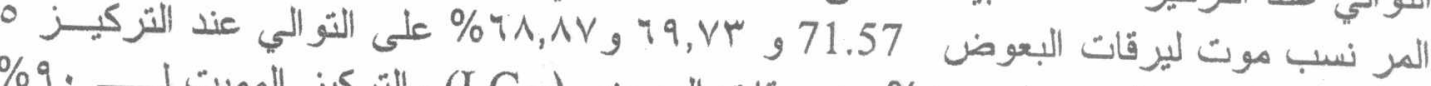

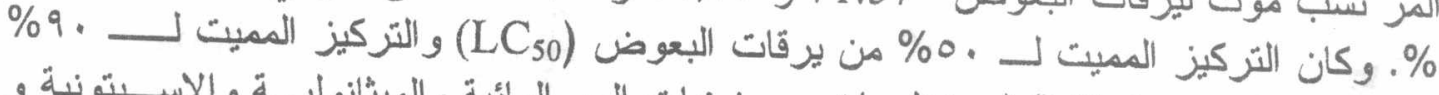

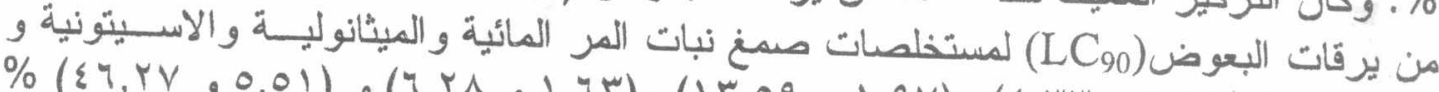

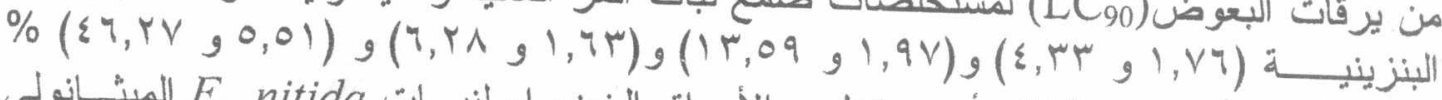

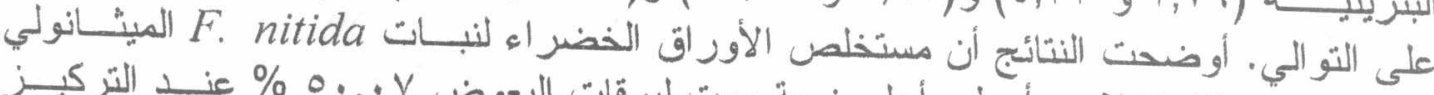

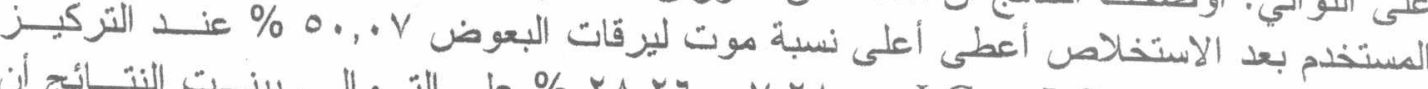

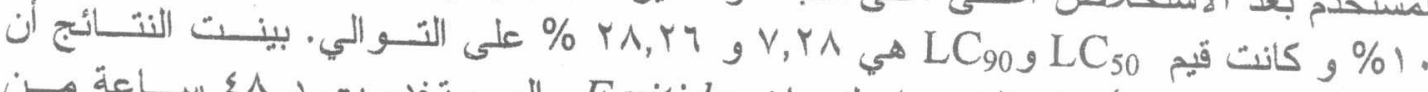

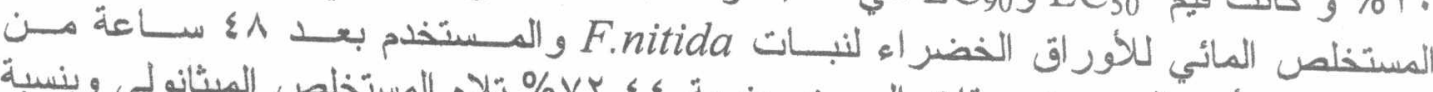

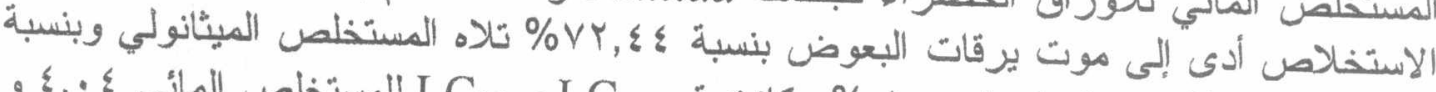

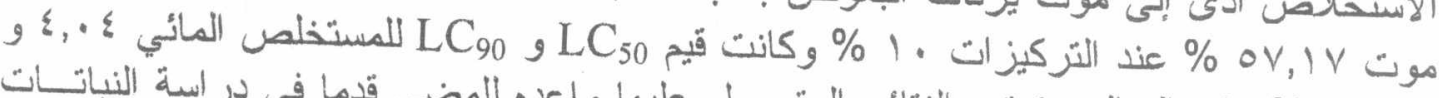

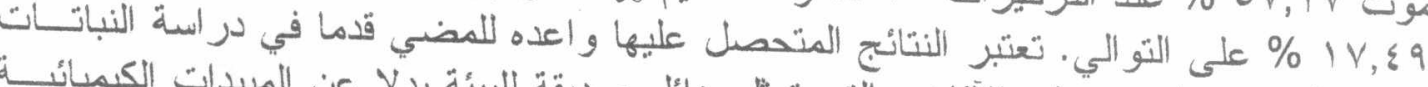

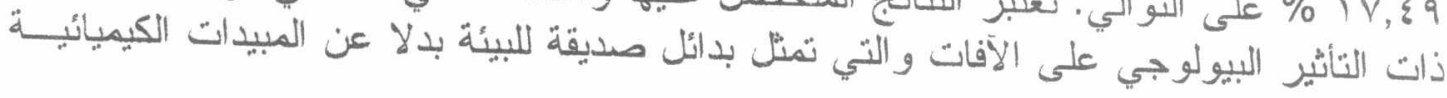

\title{
Culex quinquefasciatus Ficus nitidag
}

المقدمة:

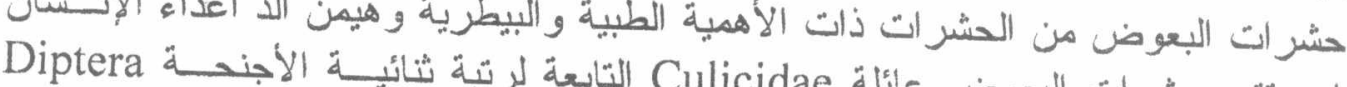

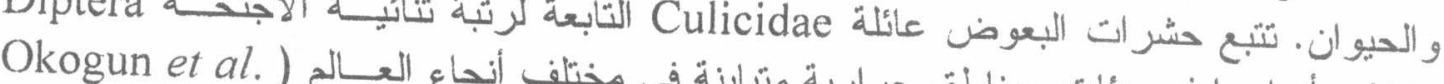

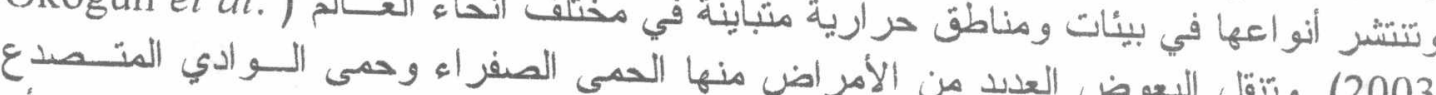

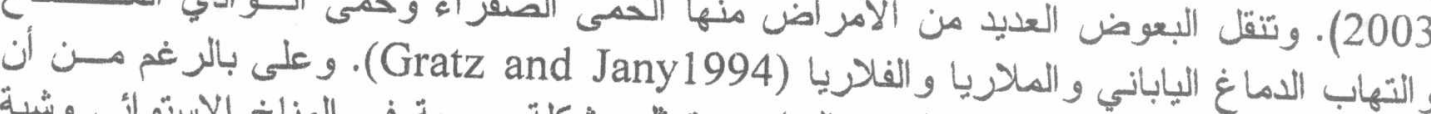

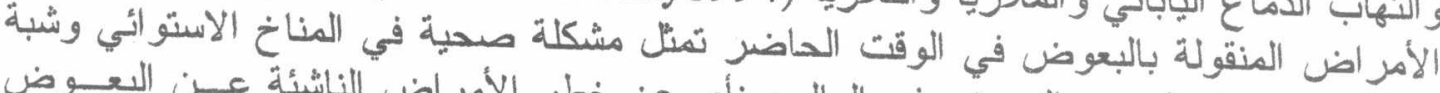

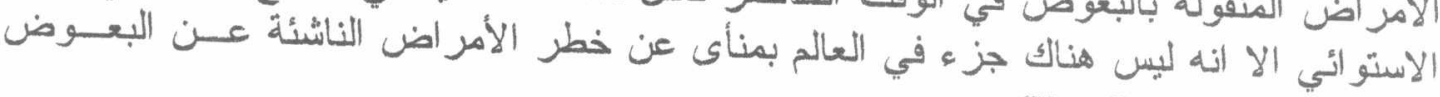

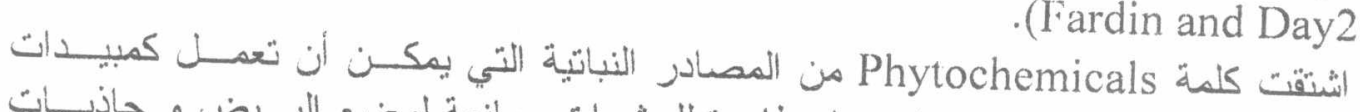

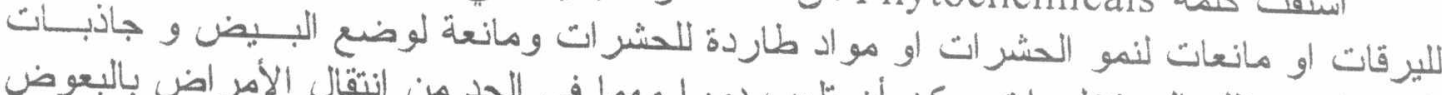

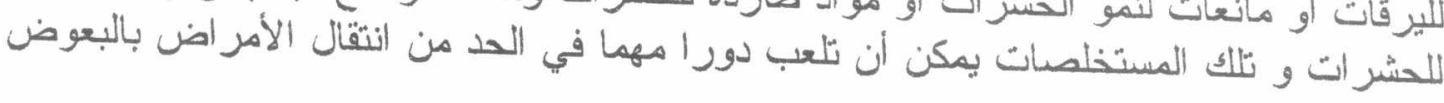


Babu and Maruugan 1998; Venketachalam and Jebasan 2001 a\&b; Mittal ) and Subbarao 2003; Bagavan et al. 2008; Ghosh et al. 2008; Kannathasan (et al. 2008; Rahuman et al.2008; Senthilkumar et al. 2008.

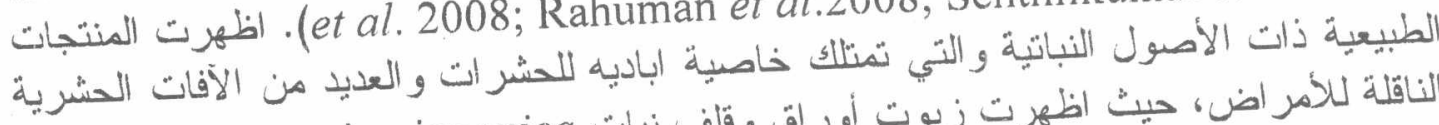

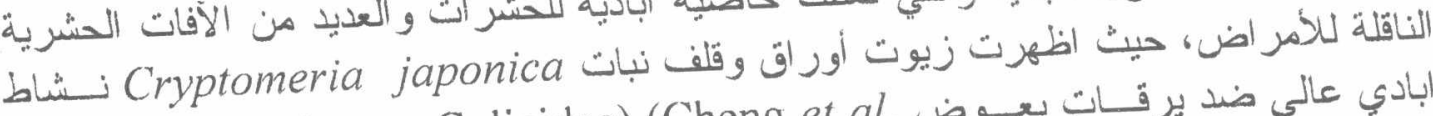

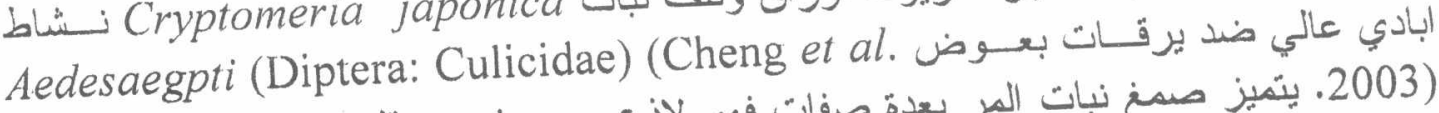

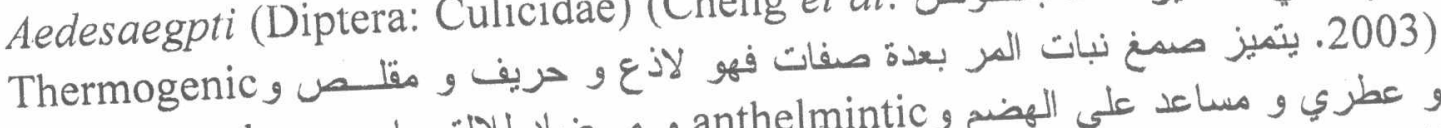

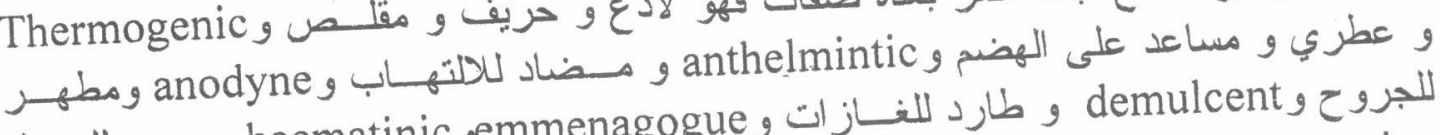

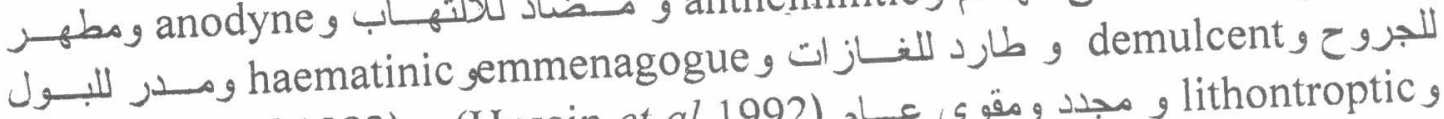

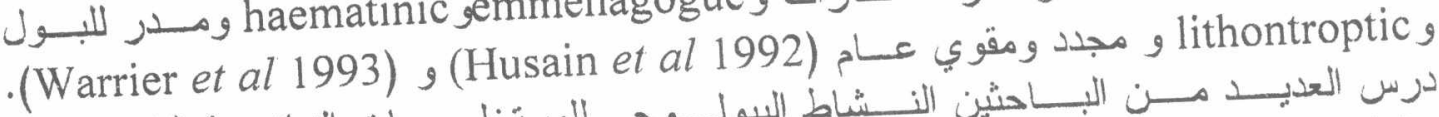

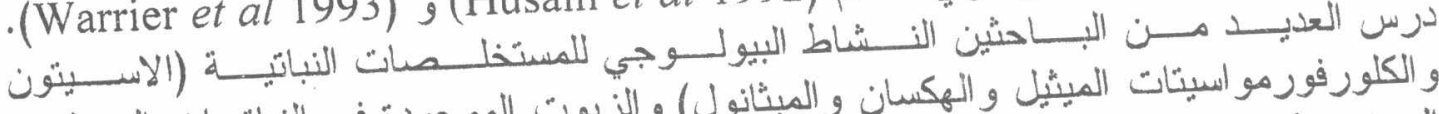

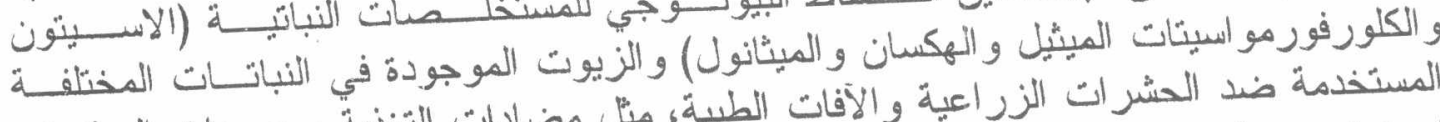

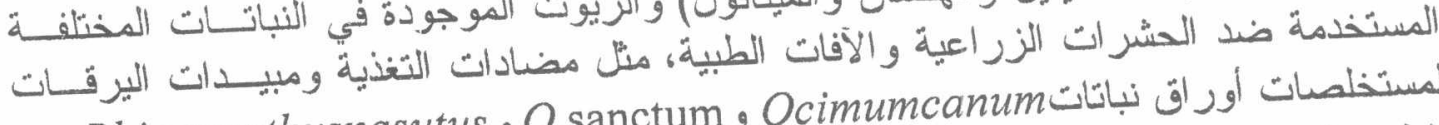

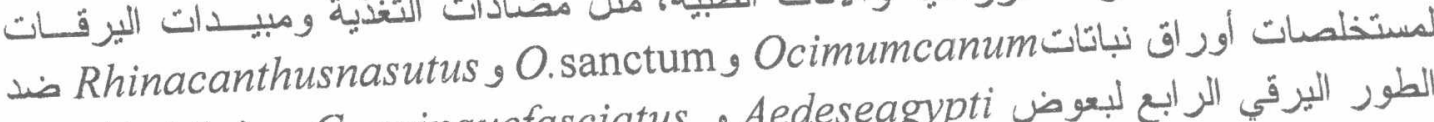
الطور البرفي الر ابع لبعوض C. quinquefasciatus و Aedeseagypti Morimoto et al. 2002; koul et al.2003; Rahuman et al. 2008; ) إلى ان تأثنبر ها معتـدن (Kamaraj et al.2008; Amer and Mehlhorn, H. 2006 a\&b

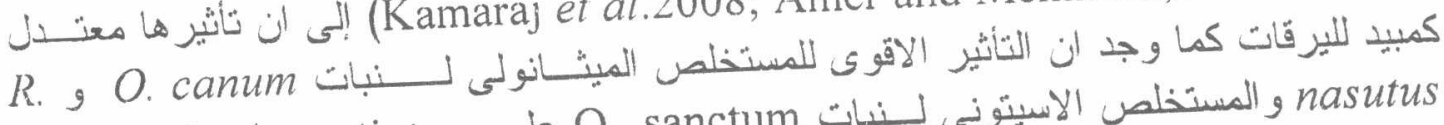

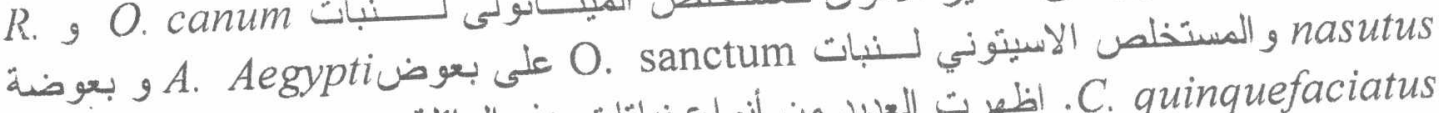

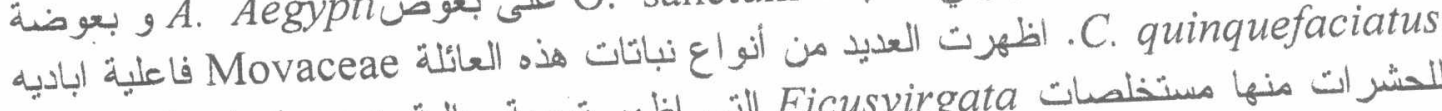

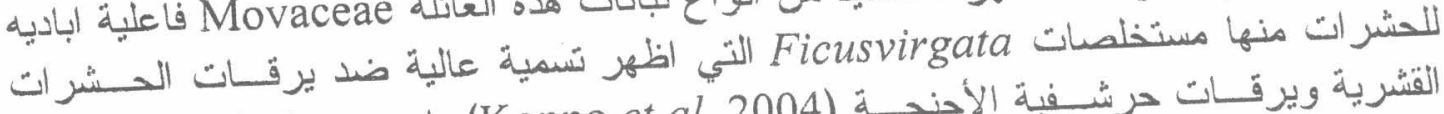

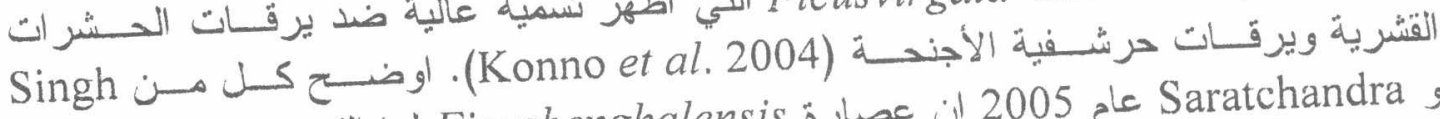

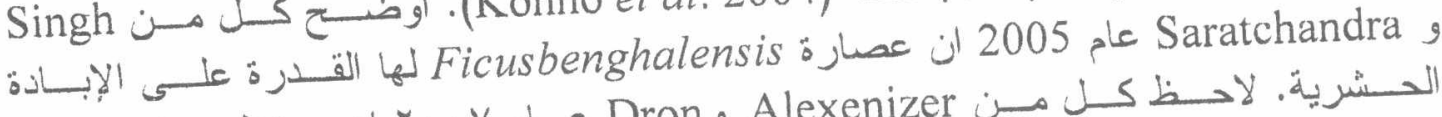

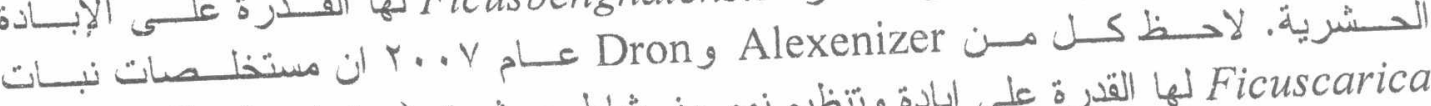

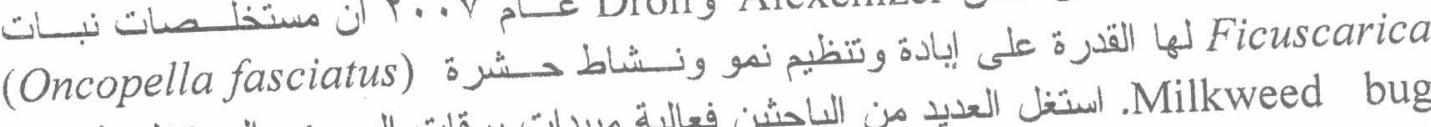
Milkweed bug

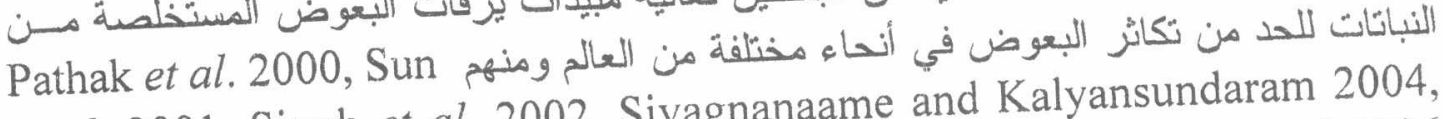
et al. 2001, Singh et al. 2002, Sivagnanaame and Kalyansundaram 2004, فأ في در اسة لـ Obomanu et al. 2006; Singh et al 2006

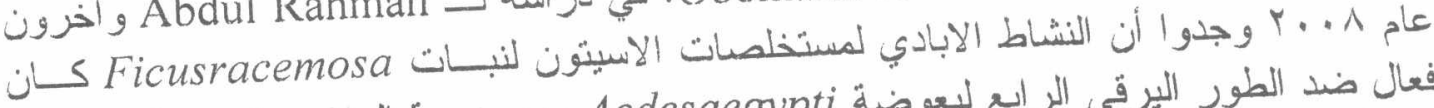

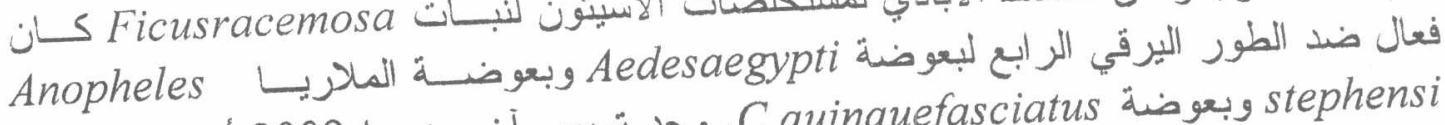
stephensi

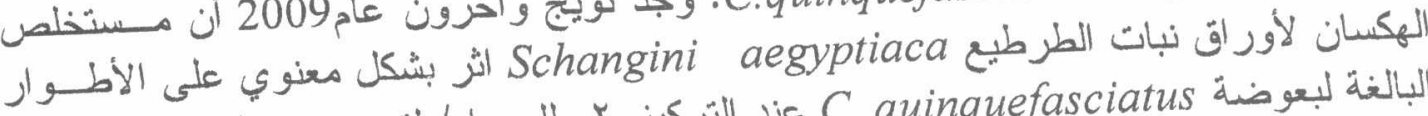

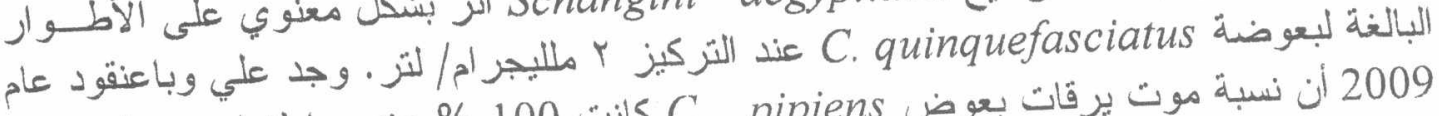

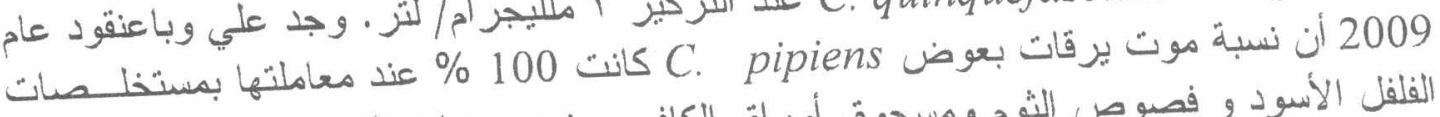

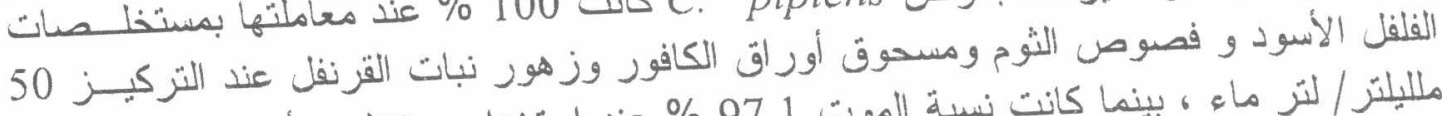

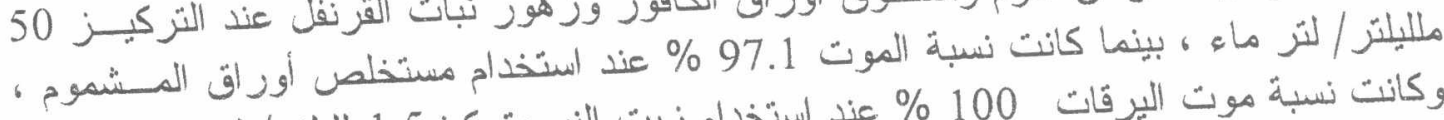

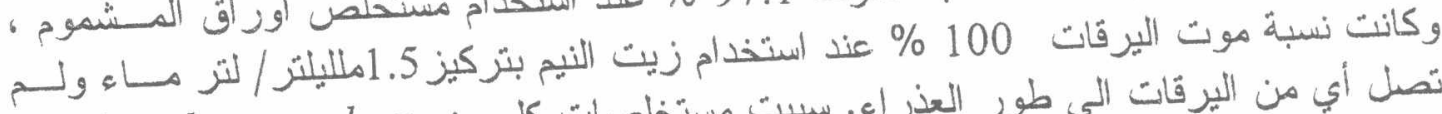
تصل أي من اليرقات الى طور العذر اء. سببت مستخلصات كل من 
Quercus infectoria DL. و Cleome glaucescens Dc. و Jack

C.pipiens

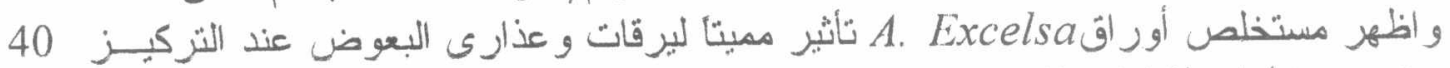

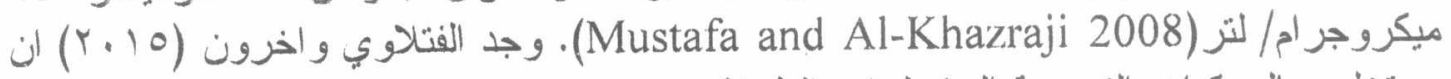

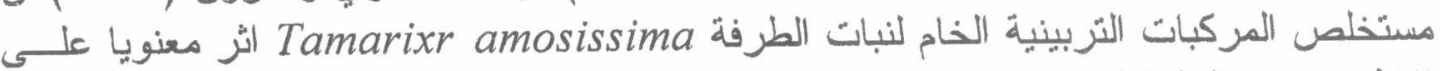
الاطو ار غير البالغة لحشرة البعوض C. pipienes حيث بلغت نسبة الاهلاك للبيض و اليرقات

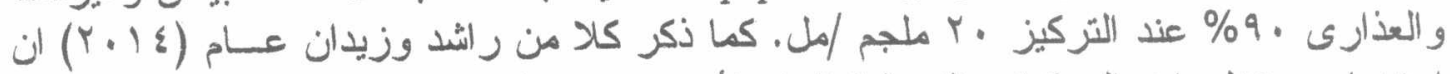

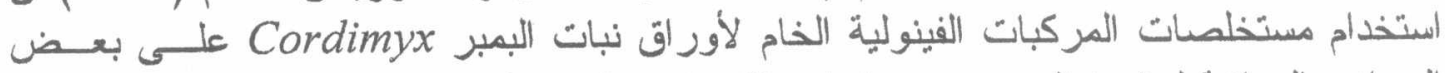

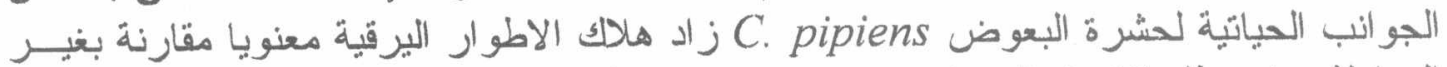

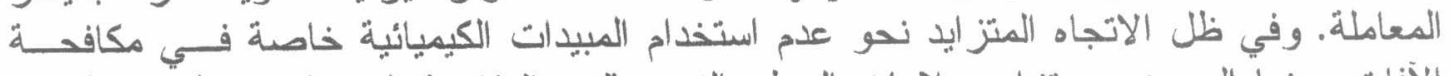

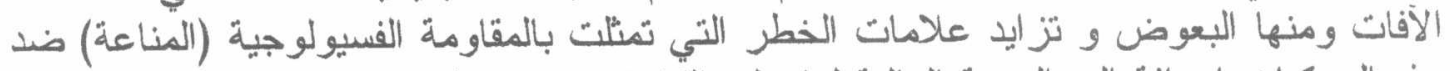

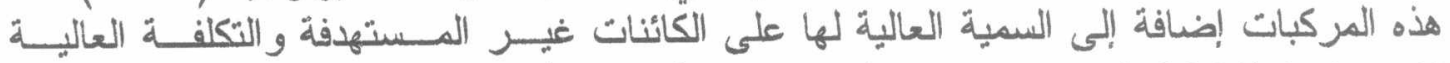

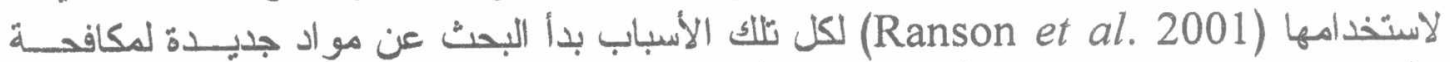

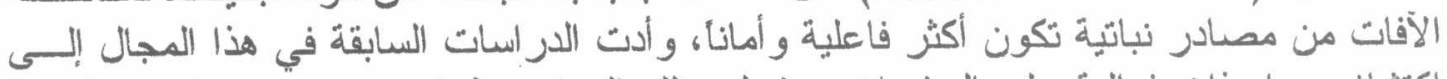

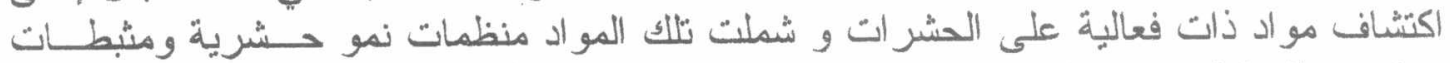

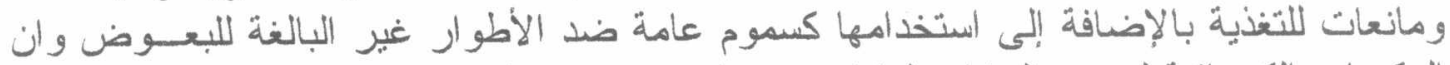

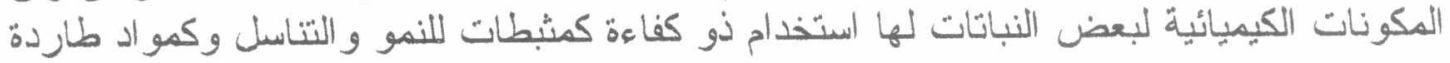

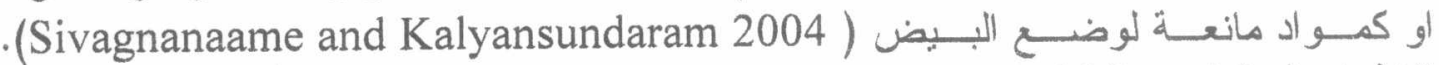

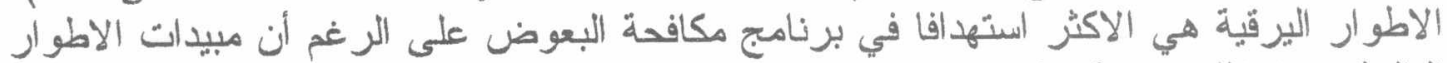

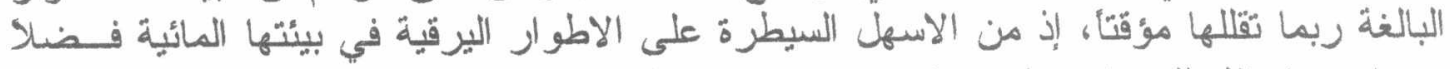

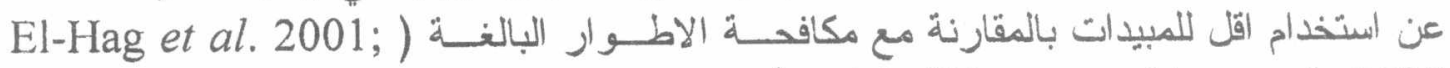
(Dharmagadda et al. 2005

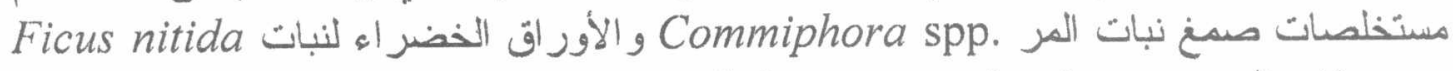
ضد يرقات البعوض Culex quinquefasciatus ندات

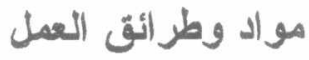

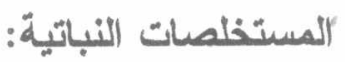

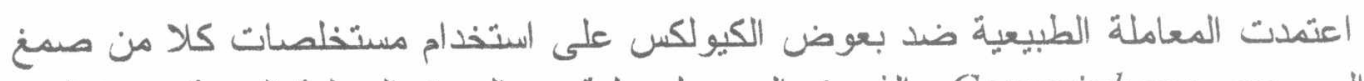

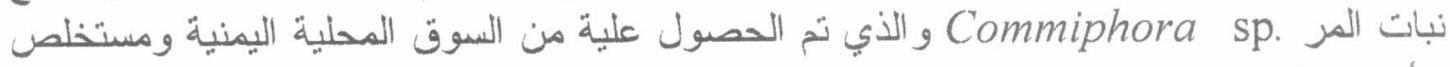

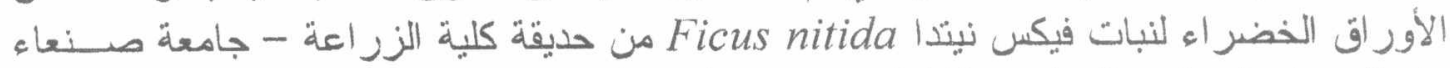

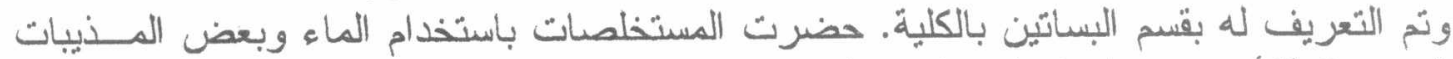

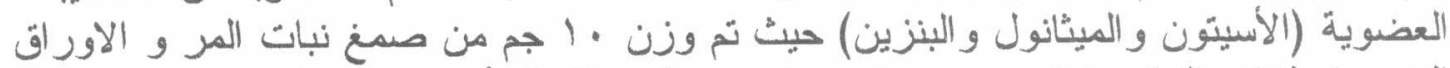

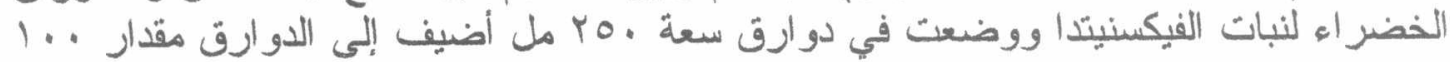

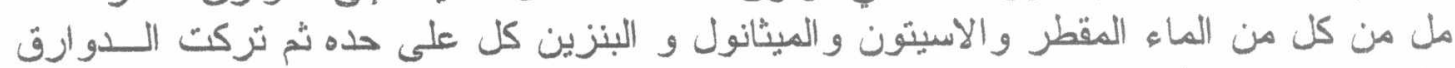

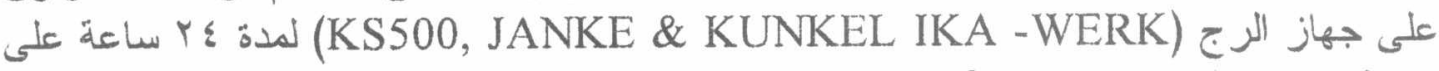
درجة حرارة المعمل 25I2C

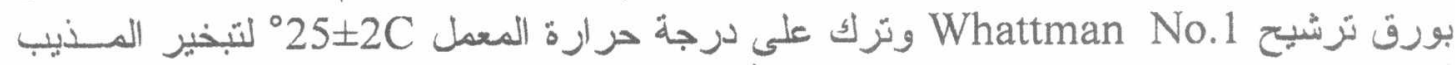

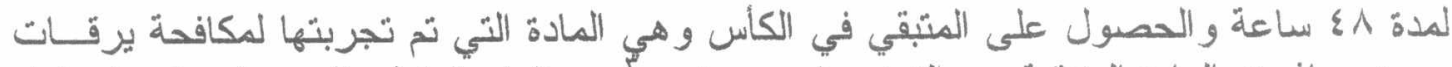

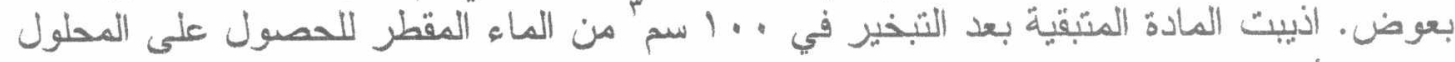

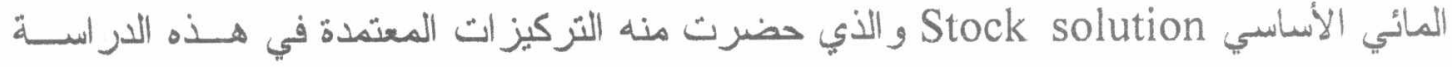




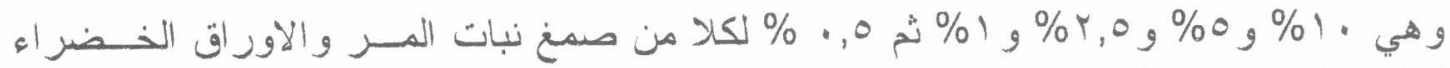

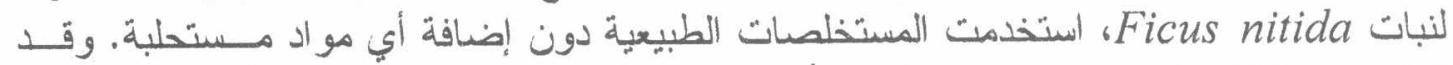

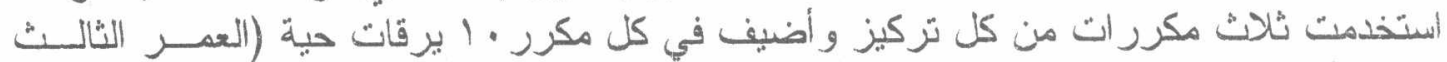

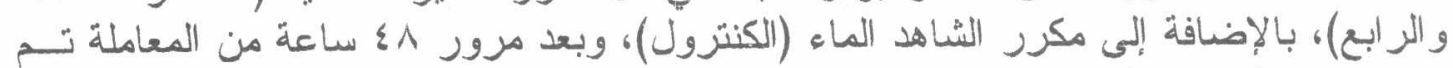
حصر عدد اليزقات المقافيتة.

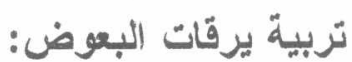

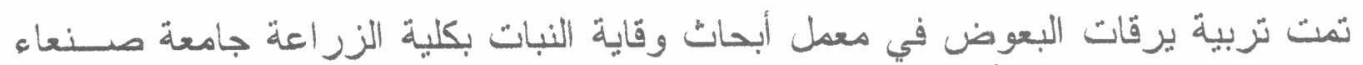

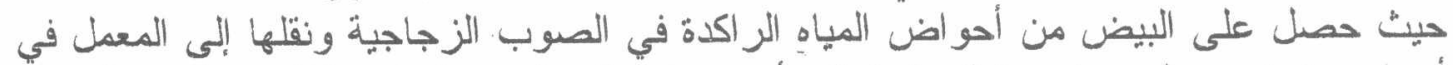

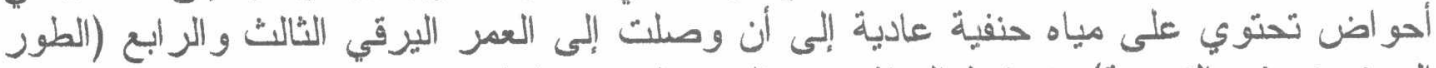

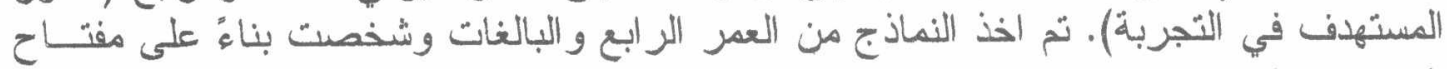

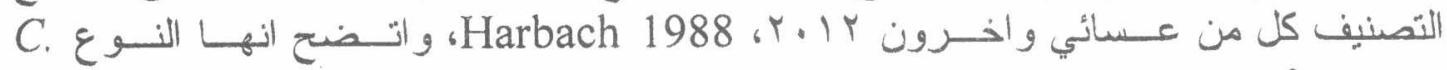
ومuinquefasciatus

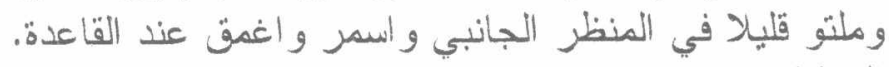
التحليل الاحصائسي:

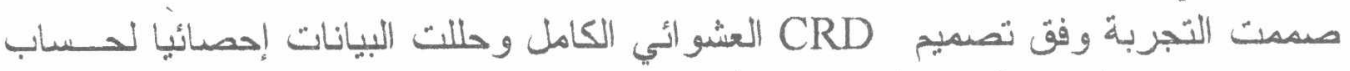

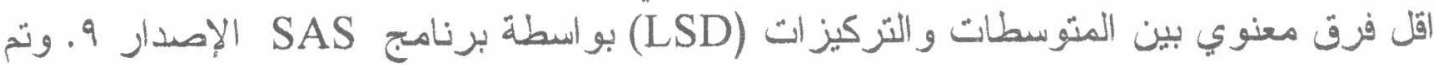

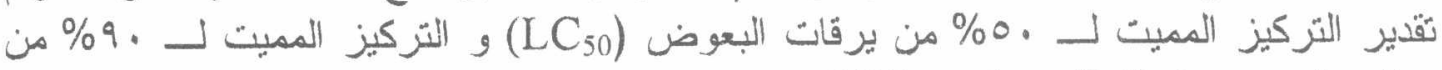

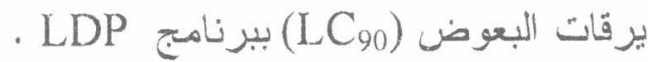

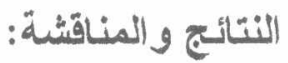
تم در اسدة تأثير المستخلص المائي و الميثانولي و الاسبيتوني و البنزيني لكل من صمغ نبات

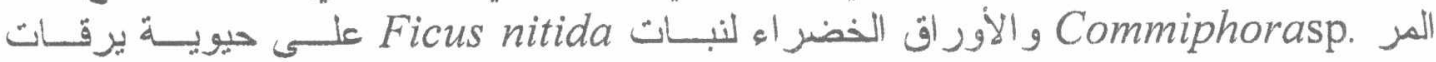

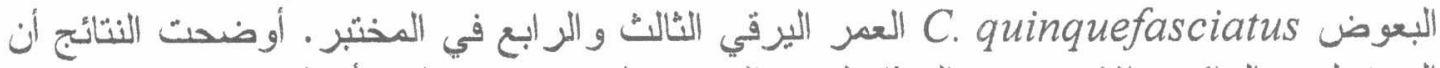

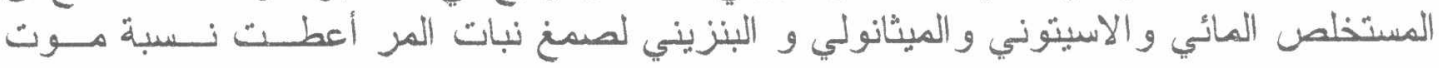

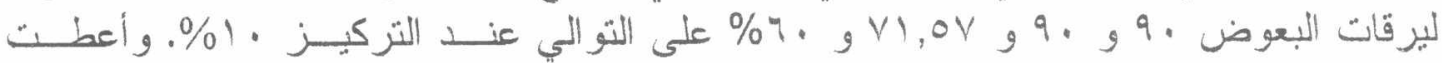

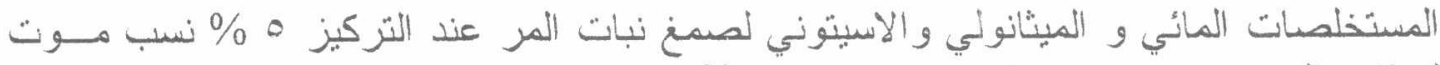

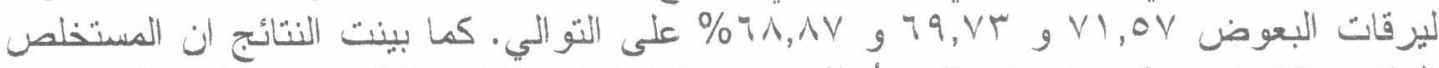

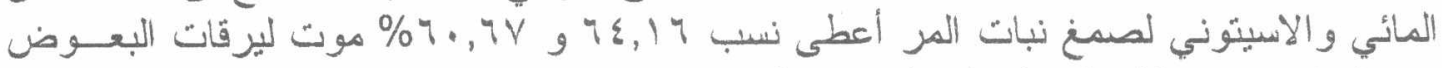

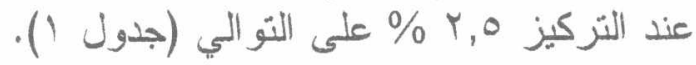

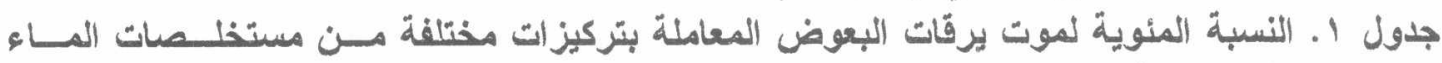

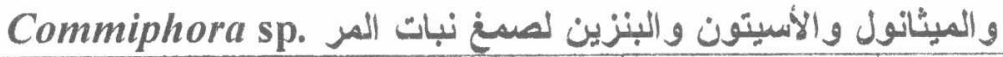

\begin{tabular}{|c|c|c|c|c|c|}
\hline \multicolumn{4}{|c|}{ النسبة المئويةٌ لموت يرقات البعوض بمستخلص } & \multirow{2}{*}{ (ماء مقطر) } & \multirow{2}{*}{ التملتوليز الاساسي هن } \\
\hline البنزين & الأسيتهن & المبئانول & 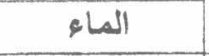 & & \\
\hline $60.00 \mathrm{~A}(\mathrm{~g})$ & $90.00 \mathrm{~A}(\mathrm{a})$ & $71.57 \mathrm{~A}(\mathrm{~b})$ & $90.00 \mathrm{~A} \mathrm{(a)}$ & $2.50 \mathrm{~A}(\mathrm{r})$ & 1. \\
\hline $37.47 \mathrm{~B}(\mathrm{~h})$ & $68.87 \mathrm{~B}(\mathrm{~d})$ & $69.73 \mathrm{~B}(\mathrm{c})$ & $71.57 \mathrm{~B}(\mathrm{~b})$ & $2.50 \mathrm{~A}(\mathrm{r})$ & $\bullet$ \\
\hline $34.45 \mathrm{C}(\mathrm{j})$ & $60.67 \mathrm{C}(\mathrm{f})$ & $30.66 \mathrm{C}(\mathrm{l})$ & $64.16 \mathrm{C}(\mathrm{e})$ & $2.50 \mathrm{~A}(\mathrm{r})$ & Y.o \\
\hline $21.56 \mathrm{D}(\mathrm{n})$ & $26.57 \mathrm{D}(\mathrm{m})$ & $35.97 \mathrm{D}(\mathrm{i})$ & $21.22 \mathrm{D}(\mathrm{o})$ & $2.50 \mathrm{~A}(\mathrm{r})$ & 1 \\
\hline $15.00 \mathrm{E}(\mathrm{q})$ & $18.43 \mathrm{E}(\mathrm{p})$ & $31.11 \mathrm{E}(\mathrm{k})$ & $2.50 \mathrm{E}(\mathrm{r})$ & $2.50 \mathrm{~A}(\mathrm{r})$ & $\because 0$ \\
\hline \multicolumn{5}{|c|}{7.0072} & LSD \\
\hline 5.51 & 1.63 & 1.97 & 1.76 & & $\mathrm{LC}_{50}$ \\
\hline 46.27 & 6.28 & 13.59 & 4.33 & & $\mathrm{LC}_{90}$ \\
\hline
\end{tabular}




\section{المتوسطات ذات الحرف الواحد لا توجد فروق معنوية بينهم حسب اختبار اقل فرق معنوي.}

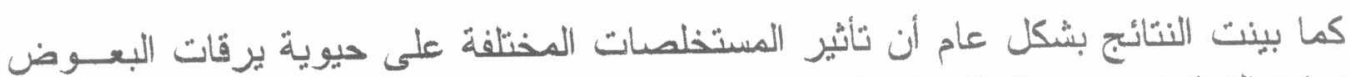

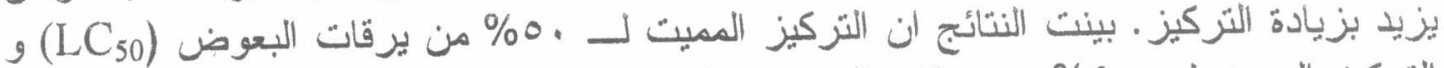

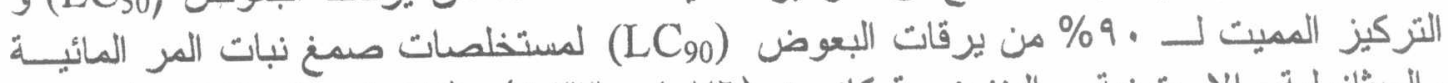

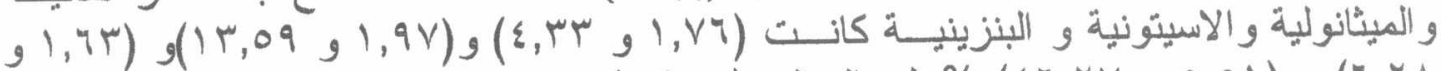

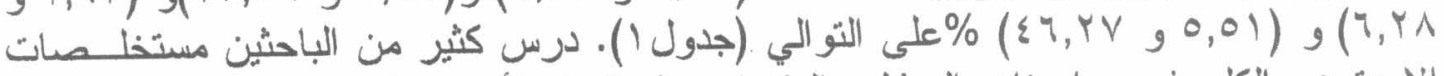

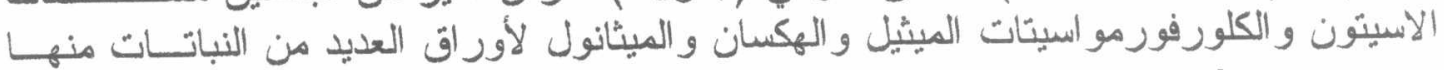
Ocimum canum, Ocimum sanctum, Rhinacanthus nasutus لبرفات بعوض C. quinquefasciatus و. Aedeseagypti. و أظهرت النتائج أن هناك نأثير

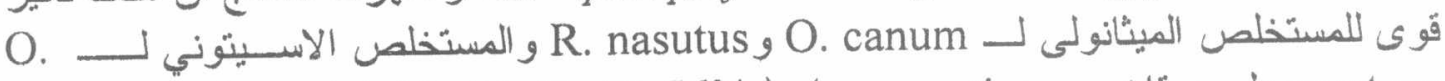
على يرقات بعوض sanctum بعوضة C. quinquefaciatus ) (Morimoto et al. 2002; koul et al. 2003; Mehlhorn and Amer 2006 a\&b; Rahuman et al. 2008a, Kamaraj et al.2008).

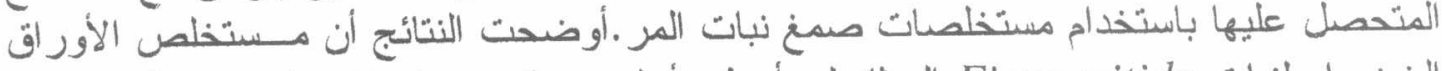

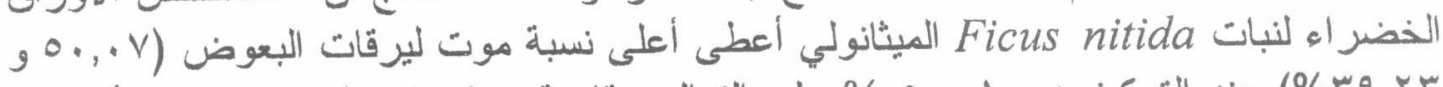

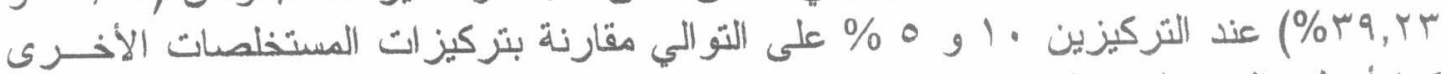

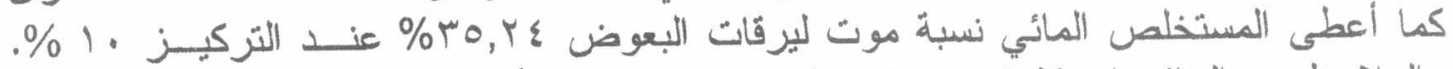

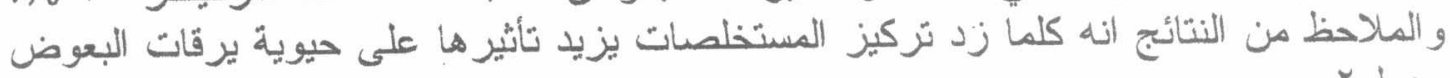

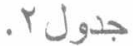

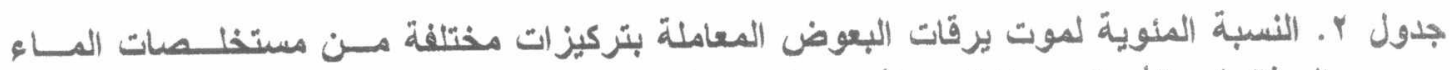

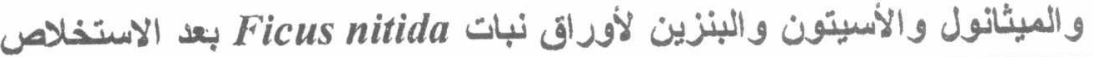

\begin{tabular}{|c|c|c|c|c|c|}
\hline \multicolumn{4}{|c|}{ النسبة المنوية لموت برقات البعوض بمستخلص } & \multirow{2}{*}{ (ماء هقطر) } & \multirow{2}{*}{ \% الأسناسي المحلول } \\
\hline البنزين & الأسبيتون & 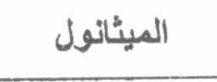 & الماء & & \\
\hline $13.18 \mathrm{~A}(\mathrm{~h})$ & $15.00 \mathrm{~A}(\mathrm{e})$ & $50.07 \mathrm{~A}(\mathrm{a})$ & $35.24 \mathrm{~A}(\mathrm{c})$ & $2.50 \mathrm{~A}(1)$ & 1 \\
\hline $12.92 \mathrm{~B}(\mathrm{j})$ & $13.94 \mathrm{~B}(\mathrm{~g})$ & $39.23 \mathrm{~B}(\mathrm{~b})$ & $23.11 \mathrm{~B}(\mathrm{~d})$ & $2.50 \mathrm{~A}(1)$ & 0 \\
\hline $2.50 \mathrm{C}(1)$ & $7.49 \mathrm{C}(\mathrm{k})$ & $24.12 \mathrm{C}(\mathrm{g})$ & $14.06 \mathrm{C}(\mathrm{f})$ & $2.50 \mathrm{~A}(1)$ & r.o \\
\hline $2.50 \mathrm{C}(1)$ & $2.50 \mathrm{D}(1)$ & $2.50 \mathrm{D}(1)$ & $2.50 \mathrm{D}(1)$ & $2.50 \mathrm{~A}(\mathrm{l})$ & 1 \\
\hline $2.50 \mathrm{C}(1)$ & $2.50 \mathrm{D}(1)$ & $2.50 \mathrm{D}(1)$ & $2.50 \mathrm{D}(1)$ & $2.50 \mathrm{~A}(1)$ & $\because 0$ \\
\hline \multicolumn{5}{|c|}{4.628} & LSD \\
\hline 2490.69 & 803.34 & 7.28 & 18.79 & & $\mathrm{LC}_{50}$ \\
\hline 20753.58 & 37153.45 & 28.26 & 105.14 & & $\mathrm{LC}_{90}$ \\
\hline
\end{tabular}

المتوسطات ذات الحرف الو احد لا توجد فروق معنوية بينهم حسب اختبار اقل فرق معنوي.

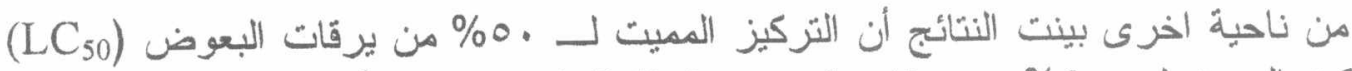

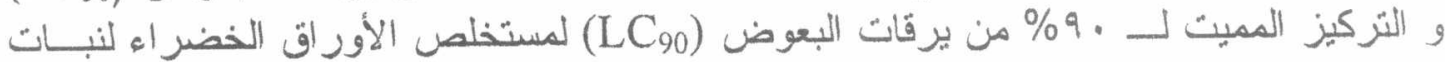

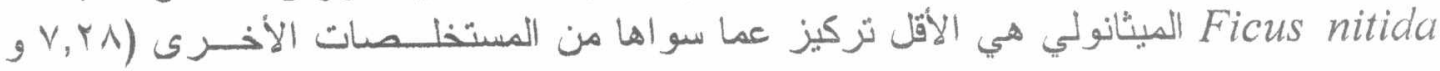




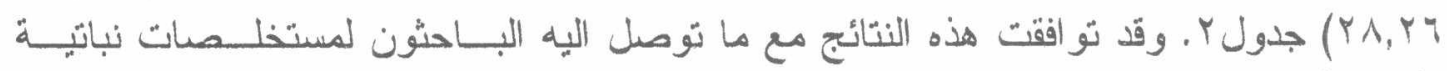

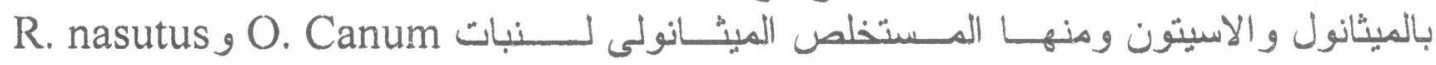

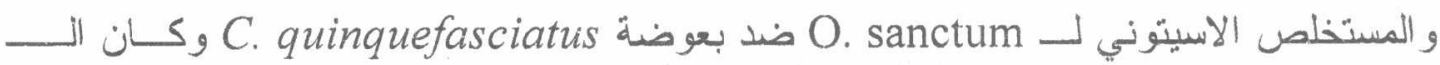
. على التو الي (Kamarj, et al., 2008 44.36, 73.40, 38.30

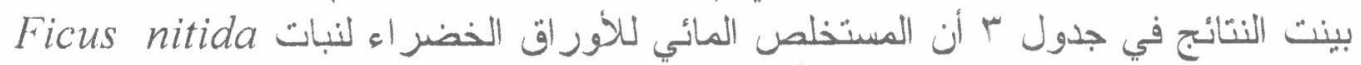

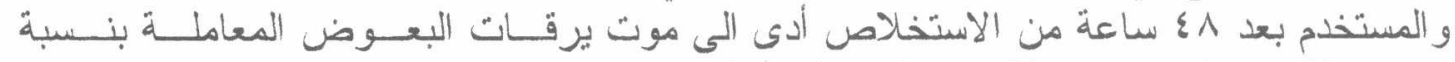

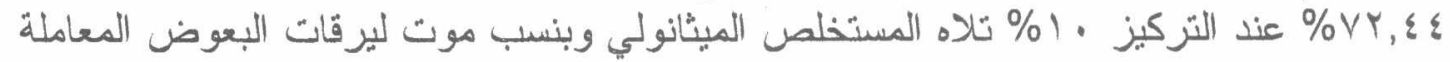

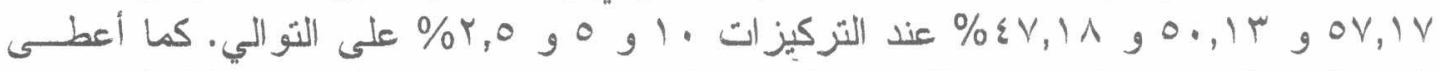

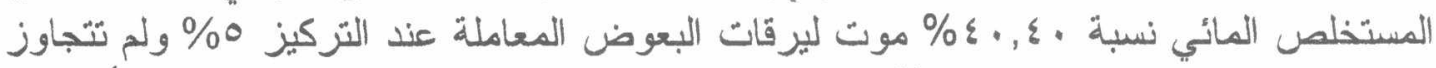

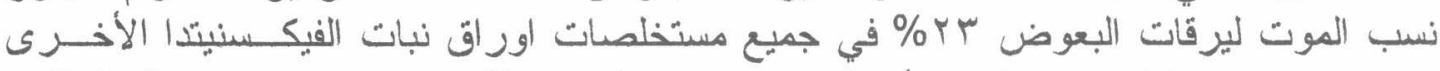

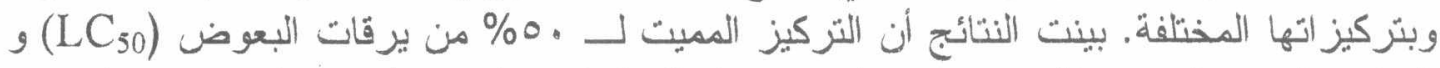

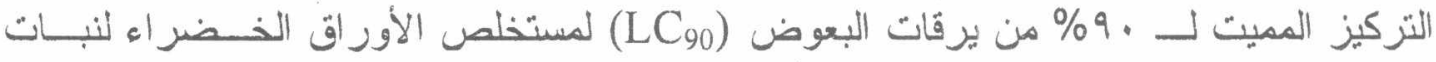
Ficus nitida

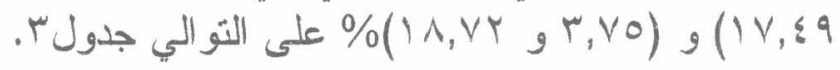

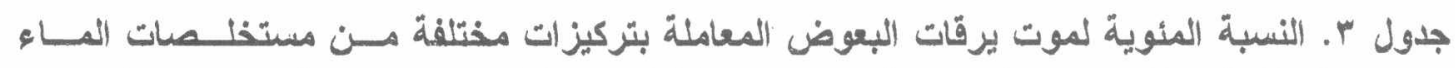

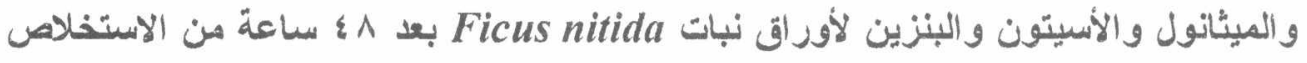

\begin{tabular}{|c|c|c|c|c|c|}
\hline \multicolumn{4}{|c|}{ النسبة المذوية لموت برقات البعوض بمستخلصات نبات المر } & \multirow{2}{*}{ (ماء مقطر) } & \multirow{2}{*}{ \% من المحلول الايساسيز } \\
\hline 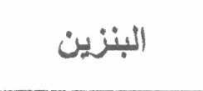 & الأسيتون & 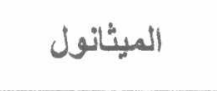 & 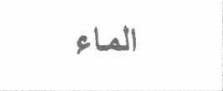 & & \\
\hline $22.79 \mathrm{~A}(\mathrm{f})$ & $21.39 \mathrm{~A}(\mathrm{~g})$ & $57.17 \mathrm{~A}(\mathrm{~b})$ & $72.44 \mathrm{~A} \mathrm{(a)}$ & $2.50 \mathrm{~A}(\mathrm{n})$ & 1. \\
\hline $18.63 \mathrm{~B}(\mathrm{k})$ & $20.00 \mathrm{~B}(\mathrm{j})$ & $50.13 \mathrm{~B}(\mathrm{c})$ & $40.40 \mathrm{~B}(\mathrm{e})$ & $2.50 \mathrm{~A}(\mathrm{n})$ & $\circ$ \\
\hline $14.30 \mathrm{C}(\mathrm{l})$ & $10.14 \mathrm{C}(\mathrm{m})$ & $47.18 \mathrm{C}(\mathrm{d})$ & $29.93 \mathrm{C}(\mathrm{f})$ & $2.50 \mathrm{~A}(\mathrm{n})$ & Y.o \\
\hline $2.50 \mathrm{D}(\mathrm{n})$ & $2.50 \mathrm{D}(\mathrm{n})$ & $20.53 \mathrm{D}(\mathrm{h})$ & $28.73 \mathrm{D}(\mathrm{g})$ & $2.50 \mathrm{~A}(\mathrm{n})$ & 1 \\
\hline $2.50 \mathrm{D}(\mathrm{n})$ & $2.50 \mathrm{D}(\mathrm{n})$ & $2.50 \mathrm{E}(\mathrm{n})$ & $2.50 E(n)$ & $2.50 \mathrm{~A}(\mathrm{n})$ & $\because 0$ \\
\hline \multicolumn{5}{|c|}{6.936} & LSD \\
\hline 84.77 & 85.89 & 3.75 & 4.04 & & $\mathrm{LC}_{50}$ \\
\hline 1337.55 & 1225.64 & 18.72 & 17.49 & & $\mathrm{LC}_{90}$ \\
\hline
\end{tabular}

المتوسطات ذات الحرف الواحد لا توجد فروق معنوية بينهو حسب اختبار اقل فرق معنوي.

وكان المستخلص الاسيتوني و البنزيني لأور اق نبات Ficus nitida الأقل نـأثير ا علــى

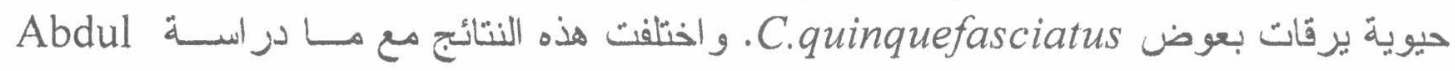

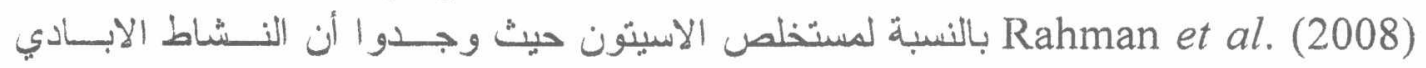
لمستخلصات الاسبتون لبنات Ficus racemosa كان فعال ضد الطور الر ابع ليرقات البعوض البون حبث كان

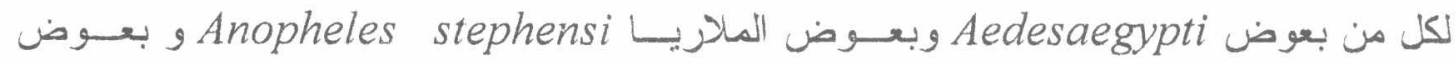
Culex quinquefasciatus في جزئية تأثير المستخلصات المائية و الميثانونية لأوراق نبات Ficus nitida علــى ميويسة مئسة 


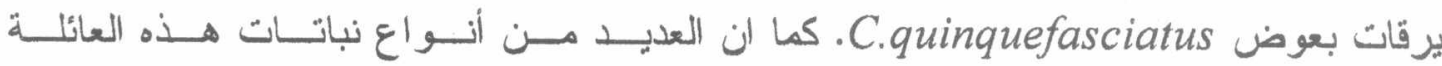
Movaceae Ficusvirgata

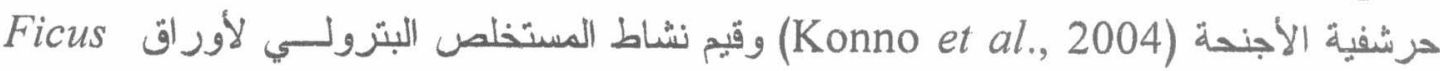

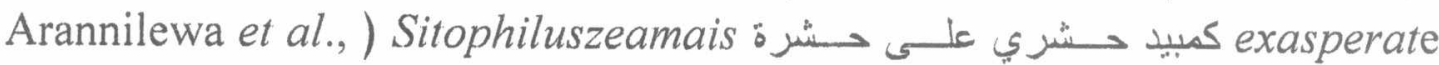
2006) كما وجد ان عصارة Ficus benghalensis) لها القدرة على الإبادة الحشرية Singh) and Saratchandra 2005) ولوحظ أن مستخلصات نبات Ficuscarica

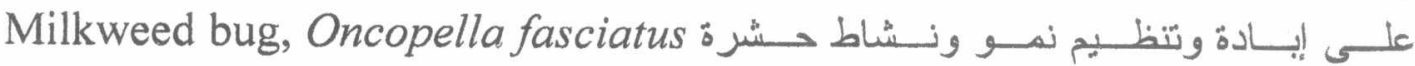

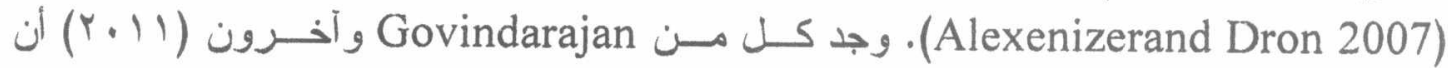

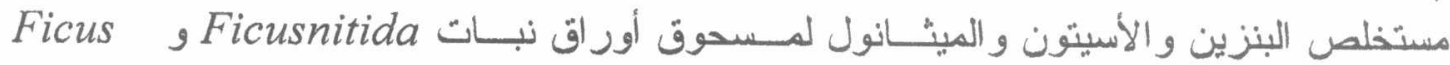

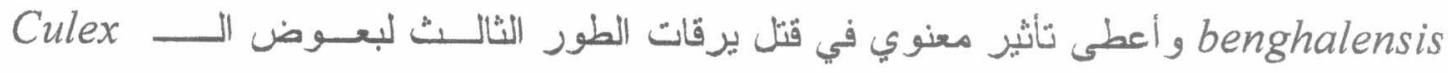
$\mathrm{LC}_{90}, \mathrm{LC}_{50}$ Anopheles subpictus وبــ tritaeniorhynchus

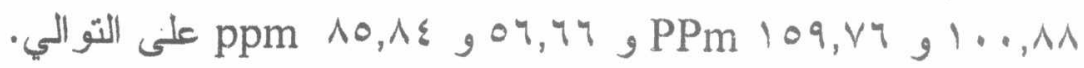

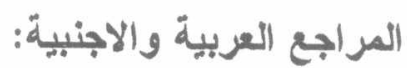

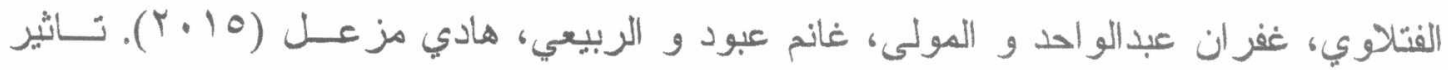

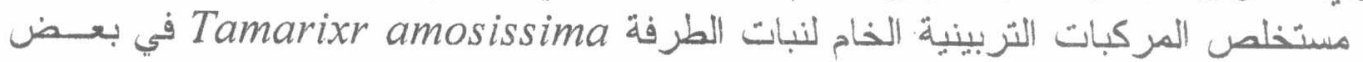

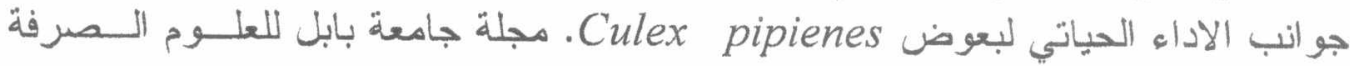

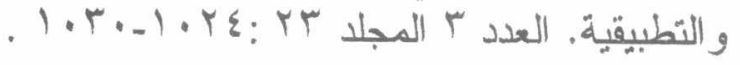

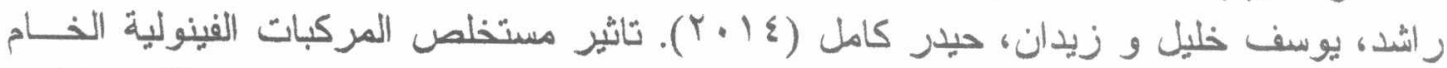

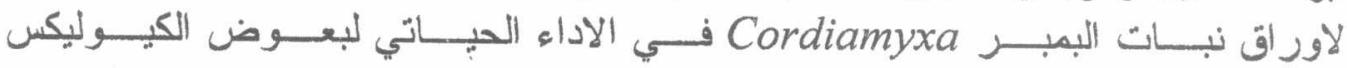
لإني

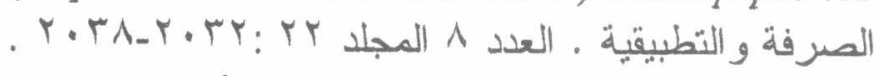

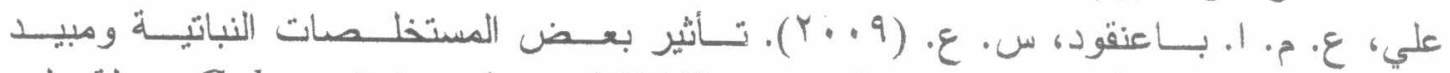

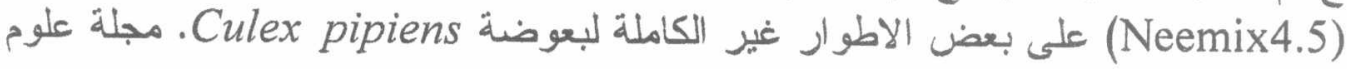

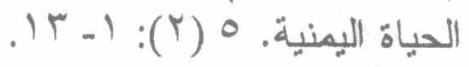

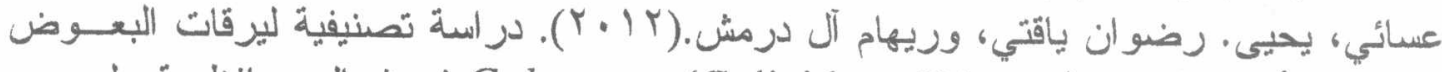

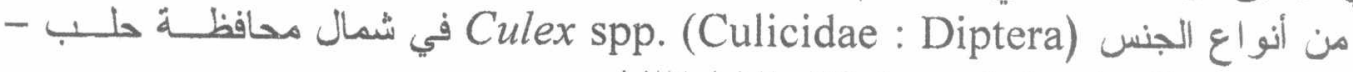

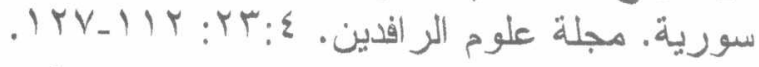

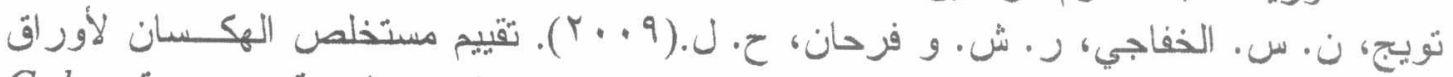

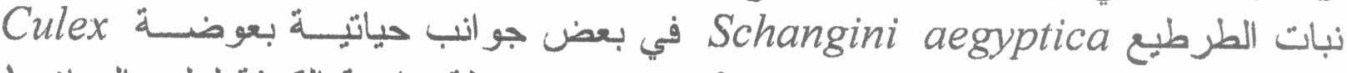
.quinquefasciatus (diptera:Culicidae) Say. $.7-1)$

Abdul Rahuman A.; Venkatesan, P.; Geetha K.; Gopalakrishnan G.; Bagavan, A. and Kamaraj, C. (2008). Mosquito larvicidal activity of gluanol acetate, a tetracyclic triterpenes derived from Ficusracemosa Linn. Parasitol Res.103:333-339. 
Alexenizer M, Dorn A (2007). Screening of medicinal and ornamental plants for insecticidal and growth regulating activity. J. Pest Sci 80:205-215.

Amer, A. and Mehlhorn H. (2006a). Larvicidal effects of various essential oils against Aedes, Anopheles, and Culex larvae (Diptera, Culicidae). Parasitol Res 99:466-472.

Amer, A. and Mehlhorn H.(2006b). Persistency of larvicidal effects of plant oil extracts under different storage conditions. Parasitol Res 99:473 -477.

Arannilewa ST. Ekrakene T, Akinneye JO (2006). Laboratory evaluation of four medicinal plants as protectants against the maize weevil, Sitophiluszeamais (Mots). Afr. J. Biotechnol 5:2032-2036.

Babu R., Murugan K. (1998). Interactive effect of neem seed kernel and neem gum extract on the control of Culex quinquefasciatus Say. Neem Newsl 15(2):9-11.

Bagavan A, Rahuman AA, Kamaraj C and Geetha K (2008). Larvicidal activity of saponin from Achyranthesaspera against Aedesaegypti and Culex quinquefasciatus (Diptera: Culicidae). Parasitol Res 103(1):223-229.

Cheng S.S, Chang HT, Chang ST, Tsai KH, and Chen WJ (2003). Bioactivity of selected plant essential oils against the yellow fever mosquito Aedesaegypti larvae. BioresTechnol 89(1):99- 102.

Dharmagadda, V.S.; Naik, S.N.; Mittal, P.K.; Vasudevan, P. (2005). Larviciadalactivity of Tayetespattulaessential oil against three mosquito species. Biosource Technol., 96, 1235-1240.

El-Hag, E.A.; Abd-ElTahman, O.; El-Nadi, H.; Zaitoon, A.A. (2001). Effects of Methanolic Extracts of Neem Seeds on Egg Hatchability and Larval Development of Culex pipiens Mosquitoes. Indian Vet.J. 78, 199- 201.

Fradin MS, Day JF (2002). Comparative efficacy of insect repellents against mosquito bite. New Eng J Med 347(1):13-18.

Ghosh A., Chowdhury N., and Chandra G. (2008). Laboratory evaluation of a phytosteroid compound of mature leaves of Day Jasmine (Solanaceae: Solanales) against larvae of Culex quinquefasciatus (Diptera: Culicidae) and nontarget organisms. Parasitol Res 103(2):271-277.

Goddard L.B.; Roth A.E.; Reisen W.K. and Scott T.W. (2003). Vertical of West Nile virus by three California Culex (Diptera: Culicidae) species. Journal of Medical Entomology 40: 743-746.

Gratz NG, Jany WC (1994). What role for insecticides in vector control programs? Am J Trop Med Hyg 50(6 Suppl):11-20.

Govindarajan, M.; Sivakumar, R.; Amsath, A. and Niraimathi, S. (2011). Mosquito larvicidal properties of Ficus benghalensis L. (Family: Moraceae) against Culex tritaeniorhynchus Giles and Anopheles subpictusGrassi (Diptera: Culicidae). Asian Pacific Journal of Tropical Medicine. 505 509. 
Harbach, R, E. (1988). The Mosquitoes of the Subgenus Culex in Southwestern Asia and Egypt (Diptera: Culicidae). Contribution of the American Entomological Institute. 24,(1).

Husain, A., Virmani, O. P., Popli, S. P., Misra, L. N., Gupta, M. M., Srivastava, G. N. Abraham, Z. and Singh, A. K. (1992). Dictionary of Indian Medicinal Plants. CIMAP, Lucknow, India. 546p.

Kamaraj, C.; Abdul Rahuman, A. and Bagavan, A.(2008). Antifeedant and larvicidal effects of plant extracts against Spodopteralitura (F.), Aedesaegypti L. and Culex quinquefasciatus Say. Parasitol Res. 103:325331.

Kannathasan K, Senthilkumar A, Venkatesalu V, and Chandrasekaran M (2008). Larvicidal activity of fatty acid methyl esters of Vitex species against Culex quinquefasciatus. Parasitol Res 103(4):999-1001.

Konno K, Hirayama C, Nakamura M, Tateishi K, Tamura Y, Hattori M,and Kohno K (2004). Papain protects papaya trees from herbivorous insects: role of cysteine proteases in latex. Plant J 37:370-378.

Koul O, Daniewski WM, Multani JS, Gumulka M, and Singh G (2003). Antifeedants effects of the Limonoids from Entandrophragmacandolei (Meliaceae) on the gram pod borer, Helicoverpaarmigera (Lepidoptera: Noctuidae). J Agric Food Chem 51:7271-7275.

Mittal PK, and Subbarao SK (2003). Prospects in using herbal products in the control of mosquito vectors. ICMR Bulletin 33(1):1-12.

Morimoto M., Tanimoto K., Sakatani A., and Komai K. (2002). Antifeedant activity of an anthraquinone aldehyde in Galiumaparine L. against Spodopteralitura F. Phytochemistry 60:163-166.

Mustafa, M. A. and Al-Khazraji, A. (2008). Effect of some plant extracts on the Culex pipiensmulestus Forskal. larvae. Iraq. J. Vet. Sci., 22(1) 9-12.

Obomanu F.G.; Ogbalu O.K.; Gabriel U.U.; Fekarurhobo G.K. and Adediran B. I. (2006). Larvicidal properties of Lepidagathisalopecuroides and Azadirachta indica on Anopheles gambiae and Culex quinquefasciatus. Afr. J. Biotechnol. 5 (9): 761-765.

Okogun G.R.; Nwoke B.; Okere A.; Anosike J. and Esekhegbe A. (2003). Epideminological implications of preferences of breeding sites of mosquito species in Midwestern Nigeria. AnnAgric Environ med.; 10 (2): 217-222.

Pathak N.; Mittal P.K.; Singh O.P.; Vidya Sagar and Vasudevan P. (2000). Larvicidal action of essential oils from plants against the vector mosquitoes Anopheles stephensi (Liston), Culex quinquefasciatus (Say) and Aedesaegypti (L). Int. Pest Cont. 42: 53-58.

Rahuman A.A, Gopalakrishnan G, Venkatesan P, Geetha K (2008). Larvicidal activity of some Euphorbiaceae plant extracts against Aedesaegypti and Culex quinquefasciatus (Diptera: Culicidae). Parasitol Res 102:867-873.

Ranson H.;Rossiter L.; Ortelli F.; Jensen B.; Wang X.; Roth C.W., Collins F.H. and Hemingway J. (2001). Identification of a novel class of insect glu- 
tathione S-transferases involved in resistance to DDT in the malaria vector Anopheles gambiae. Biochemical Journal.; 359:295-304.

Senthilkumar A, Kannathasan K, and Venkatesalu V (2008). Chemical constituents and larvicidal property of the essential oil of Blumeamollis (D. Don) Merr against Culex quinquefasciatus. Parasitol Res 103(4):959-962.

Singh R.K.; Dhiman R.C. and Mittal P.K. (2006). Mosquito larvicidalproperties of Momordica charantia Linn (Family: Cucurbitaceae), J.Vect. Borne. Dis. 43: 88-91.

Singh RN, and Saratchandra B (2005). The development of botanical products with special reference to seri-ecosystem. Caspian J Env Sci 3:1-8.

Singh S.P.; Raghavendra K.; Singh, R.K. and Subbarao S.K. (2002). Studies on larvicidal properties of leaf extract of Solanum nigrum Linn. (Family: Solanaceae). Curr. Sci. 81: 1529-1536.

Sivagnaname N. and Kalyanasundaram, M. (2004). Laboratory evaluation of methanolic extract of Atlantia monophylla (Family: Rutaceae) against immature stages of mosquitoes and non-target organisams. Mem. Inst. Oswaldo Cruz, Rio de Janeiro. 99 (1): 115-118.

Sun R.; Sacalis J.N.; Chin C.K. and Still C.C. (2001). Bioactive aromatic compounds from leaves and stems of Vanilla fragrans, J. Agric. Food Chem., 49: 5161.

Venketachalam MR, Jebasan A (2001a). Repellent activity of Ferronia elephantum Corr. (Rutaceae) leaf extract against Aedes aegypti. Biores Technol 76(3):287-288.

Warrier, P. K., Nambiar, V. P. K. and Ramankutty, C. (1993-1995). Indian Medicinal Plants. Orient Longman Ltd., Madras. Vol. 1-5.

Zinser, M.; Ramberg, F. and Willot, E. (2004). Scientific note: Culex quinquefasciatus (Diptera: Culicidae) as a potential West Nile virus vector in Tucson, Arizona: Blood meal analysis indicates feeding on both humans and birds. 3pp. Journal of Insect cience, 4:20. 
The Effect of Extracts of Commiphora sp. gum and Ficus nitida leaves on Larval Mortality of Culex quinquefasciatus under Laboratory Conditions

Khalid, SaeedAqlannaser ${ }^{1}$ and El-moflehi, Mahmoud Ali Abdallah ${ }^{2}$

'Department of Biology, Faculty of Education-Thamar, Thamar University. kh.

${ }^{2}$ Department of Plant Protection, Faculty of Agriculture, Sana'a University, Yemen

\section{Abstract:}

This study was conducted on the effect of the extracts water, methanol, acetone and benzene for each Commiphora sp. gum plant and leaves of Ficus nitida plant on the larval mortality of Culex quinquefasciatus under laboratory conditions. The data was recorded after 48 hours of treatments. The results showed that the percentages of larval mortality of Culex quinquefasciatus with the water, methanol, acetone and benzene extracts of Commiphora sp. were 90, 90, 71.57 and $60 \%$, at $10 \%$, respectively. However, water, methanol and acetone extracts of Commiphora sp. gave $71.57,69.73$ and $68.87 \%$ larval mortality of C. quinquefasciatus, at $5 \%$, respectively. The $\mathrm{LC}_{50}$ and $\mathrm{LC}_{90}$ of the water, methanol, acetone and benzene extracts of Commiphora sp. were (1.7 and 4.33), (1.97 and 13.58), (1.63 and 6.28) and (5.51 and 46.27), \%, respectively. Methanol extract of F.nitida leaves gave the highest percentage of larval mortality of C.quinquefasciatus, $50.07 \%$ at $10 \%$ after immediate extraction and the $\mathrm{LC}_{50}$ and $\mathrm{LC}_{90}$ values were 7.28 and $28.26 \%$, respectively. The results showed that the water extract of F.nitida leaves, was used after $48 \mathrm{hs}$ of extraction, gave the highest mortality percentage of C.quinquefasciatus Larvae; $72.44 \%$, followed by Methanol extract with the mortality percentage of $57.17 \%$, at $10 \%$. The $\mathrm{LC}_{50}$ and $\mathrm{LC}_{90}$ values of water extract were 4.04 and $17.49 \%$, respectively. These results were considered promising and viable for further studies to be pursued pertaining to the study on bioactive plants which represent an environmentally sound alternative for synthetic pesticides.

Keywords: Extracts, Commiphora sp. gum, Ficus nitidaleaves, larval mortality, Culex quinquefasciatus 
